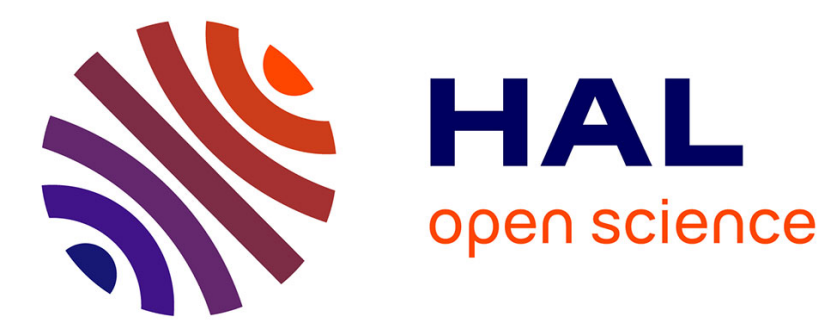

\title{
Light scattering data analysis via set inversion
}

Luc Jaulin, Jean-Luc Godet, Eric Walter, A. Elliasmine, Y. Leduff

\section{To cite this version:}

Luc Jaulin, Jean-Luc Godet, Eric Walter, A. Elliasmine, Y. Leduff. Light scattering data analysis via set inversion. Journal of Physics A: Mathematical and Theoretical, 1997, 30, pp.7733-7738. hal00843964

\section{HAL Id: hal-00843964 https://hal.science/hal-00843964}

Submitted on 15 Jul 2013

HAL is a multi-disciplinary open access archive for the deposit and dissemination of scientific research documents, whether they are published or not. The documents may come from teaching and research institutions in France or abroad, or from public or private research centers.
L'archive ouverte pluridisciplinaire $\mathbf{H A L}$, est destinée au dépôt et à la diffusion de documents scientifiques de niveau recherche, publiés ou non, émanant des établissements d'enseignement et de recherche français ou étrangers, des laboratoires publics ou privés. 


\title{
Light Scattering data analysis
}

\section{via set inversion}

\author{
L. Jaulin ${ }^{\diamond}$ J.-L. Godet*, \\ E. Walter ${ }^{\star}$, A. Elliasmine* and Y. Le Duff* \\ - Laboratoire d'Ingénierie des Systèmes Automatisés, Université d'Angers, Faculté \\ des Sciences, 2 boulevard Lavoisier, 49045 Angers, France \\ * Laboratoire des Propriétés Optiques des Matériaux et Applications, Université \\ d'Angers, Faculté des Sciences, 2 boulevard Lavoisier, 49045 Angers, France \\ * Laboratoire des Signaux et Systèmes, CNRS, Supélec, Plateau de Moulon, \\ 91192 Gif-sur-Yvette, France
}

January 8, 1999 


\begin{abstract}
Set-inversion is applied to recent collision-induced scattering data concerning gaseous $\mathrm{CF}_{4}$. It makes it possible to approximate the set of all vectors of independent components of the $\mathrm{CF}_{4}$ dipole-quadrupole and dipole-octopole polarizability tensors. Numerical analysis shows that short range effects must be taken into account in the high frequency range of each dipole-multipole contribution to the CIS isotropic spectrum of $\mathrm{CF}_{4}$. It also demonstrates the agreement between experiment and recent $a b$ initio calculations.
\end{abstract}




\section{Introduction}

In low density fluids, interactions between molecules are binary. Thus, collisioninduced light scattering (CIS) results from collisional polarizabilities of molecular pairs [1]. For optically isotropic molecules like $\mathrm{CF}_{4}$, pure collision-induced depolarized and isotropic spectra are observed in the vicinity of the Rayleigh line, where no monomolecular scattering is allowed [2]. These spectra provide information on molecular interactions and may be used to estimate the origin-independent parameters of the dipole-multipole polarizability tensors (e.g. $A$ and $E$ which characterize the dipole-quadrupole and dipole-octopole tensors $\mathbf{A}$ and $\mathbf{E}$ of any tetrahedral molecule). For pairs of molecules that cannot be easily described in terms of quantum mechanics, a semi-classical model may be considered [3]. In this case, the $\mathrm{CF}_{4}$ spectral intensity $I^{s}$ can be written as follows $[4,5]$ :

$$
\begin{aligned}
I^{s}(\omega) & =I_{D I D}^{s}(\omega)+c_{\alpha \mathrm{TA}}^{s} \alpha^{2} A^{2} I_{\alpha \mathrm{TA}}(\omega)+c_{\alpha \mathrm{TE}}^{s} \alpha^{2} E^{2} I_{\alpha \mathrm{TE}}(\omega) \\
& +c_{\mathrm{ATA}}^{s} A^{4} I_{\mathrm{ATA}}(\omega)+c_{\mathrm{ATE}}^{s} A^{2} E^{2} I_{\mathrm{ATE}}(\omega)+c_{\mathrm{ETE}}^{s} E^{4} I_{\mathrm{ETE}}(\omega)+\ldots
\end{aligned}
$$

where $\omega$ denotes the frequency shift and $\alpha$ is the main polarizability. The subscript $D I D$ refers to the dipole-induced-dipole interaction. Subscripts $\alpha \mathrm{TA}, \alpha \mathrm{TE}$, ATA, ATE and ETE refer to the successive dipole-multipole light scattering mechanisms $[4,5]$. The coefficients $c^{s}$ depend on the nature $s$ of the spectrum (depolarized 
or isotropic) and on the dipole-multipole mechanisms [4, 5]. Unfortunately, point estimation of $A$ and $E$ is highly uncertain due to the following four reasons:

i) Short range effects such as overlap and exchange effects are not taken into account in the aforementioned semi-classical model. As a result, in the $\mathrm{CF}_{4}$ case, depolarized and isotropic spectra lead to different conclusions. The isotropic spectrum has been shown to be the more adapted [5].

ii) Because of the competition between the dipole-multipole mechanisms (like $\alpha$ TA and $\alpha \mathbf{T E})$ several different parameter vectors $(A, E)$ may correspond to similar fits, i.e. the model is said to be almost unidentifiable.

iii) Errors on measurement of the isotropic spectrum are large [5] and many unsimilar fits can be considered as consistent with all data.

iv) The model is nonlinear ( $c f$. Eq. (1)) and local minimization procedures may converge to any local minimum.

For all these reasons, a bounded-error estimation approach (see [6], [7] and references therein) is considered in this work. It consists of characterizing the set $\mathcal{S}$ of all values of the vector $(A, E)$ such that the associated model output is consistent with all 
experimental data, i.e. goes through all error bars. The method to be used is new for most physicists and based on set inversion [8]. Set inversion, presented in Section 2 , is particularly suited in our case because of the nonlinearities involved in the semiclassical model; it uses interval analysis which is a numerical tool for computing with sets (also presented in Section 2). Section 3 gives an approximation of the set $\mathcal{S}$ of all feasible vectors $(A, E)$ and compares it with former estimations. We show that recent $a b$ initio computations by Maroulis $[9,10]$ partly confirm our results. Finally, we conclude on the advantages that set inversion analysis offers to spectroscopists.

\section{Set inversion}

Let $\vec{f}$ be a nonlinear continuous vector function mapping $R^{n}$ into $R^{m}$ and $\mathcal{Y}$ be a subset of $R^{m}$. The set inversion problem is to characterize the set $\mathcal{X}$ defined by

$$
\mathcal{X}=\{\vec{x} \mid \vec{f}(\vec{x}) \subset \mathcal{Y}\}=\overrightarrow{f^{-1}}(\mathcal{Y})
$$

The set function $\overrightarrow{f^{-1}}$ is the reciprocal function of $\vec{f}$. The set-inversion algorithm to be presented is based on interval arithmetic which is a numerical tool originally developed in order to quantify the effect of finite-precision arithmetic on results obtained by a computer [11]. Interval arithmetic extends classical operators on real numbers to 
intervals in a natural way. Thus, if $[x]=\left[x^{-}, x^{+}\right]$and $[y]=\left[y^{-}, y^{+}\right]$,

$$
\begin{gathered}
{[x]+[y]=\left[x^{-}+y^{-}, x^{+}+y^{+}\right],} \\
{[x]-[y]=\left[x^{-}-y^{+}, x^{+}-y^{-}\right],} \\
{[x] \cdot[y]=\left[\min \left(x^{-} y^{-}, x^{-} y^{+}, x^{+} y^{-}, x^{+} y^{+}\right), \max \left(x^{-} y^{-}, x^{-} y^{+}, x^{+} y^{-}, x^{+} y^{+}\right)\right] .}
\end{gathered}
$$

For example, we have $([1,2]+[-3,4]) \cdot[-1,5]=[-2,6] \cdot[-1,5]=[-10,30]$,

As another example, let us consider the real function $f(x)=x^{2}+2 x+4$. An interval evaluation for $f$ is $[f]([x])=[x] \cdot[x]+2[x]+4$. For $[x]=[-3,4]$, we have:

$$
[f]([-3,4])=[-3,4] \cdot[-3,4]+2[-3,4]+4=[-12,16]+[-6,8]+4=[-14,28] .
$$

Note that the actual image by $f$ of the interval $[x], f([-3,4])=[3,28]$ is a subset of the interval evaluation $[f]([-3,4])=[-14,28]$. This illustrates that interval evaluation is usually pessimistic [11].

A box or vector interval $[\vec{x}]$ of $R^{n}$ is defined as the Cartesian product of $n$ intervals.

$$
[\vec{x}]=\left[x_{1}^{-}, x_{1}^{+}\right] \times \cdots \times\left[x_{n}^{-}, x_{n}^{+}\right]
$$

The $i^{\text {th }}$ component of the box $[\vec{x}]$ is an interval denoted by $[x]_{i}$. It can be proven (see $[11])$ that the interval evaluation $[f]([\vec{x}])=[f]\left([x]_{1}, \cdots,[x]_{n}\right)$ always contains the image interval $f([\vec{x}])$, i.e.

$$
\forall[\vec{x}], f([\vec{x}]) \subset[f]([\vec{x}])
$$


The width $w([\vec{x}])$ of a box $[\vec{x}]$ is the size of its largest side. For instance, the width of the box $[\vec{x}]=[1,2] \times[-1,3]$ is equal to 4 . A principal plane of $[\vec{x}]$ is a symmetry plane of $[\vec{x}]$ normal to a side of maximum length. To bisect a box $[\vec{x}]$ means to cut it along one of its principal planes. Bisecting $[\vec{x}]=[1,2] \times[-1,3]$ produces two boxes $[\vec{x}](1)=[1,2] \times[-1,1]$ and $[\vec{x}](2)=[1,2] \times[1,3]$.

The algorithm SIVIA (Set Inverter Via Interval Analysis) partitions some prior box of interest $[\vec{x}](0)$ into a set of non-overlapping boxes. For the sake of simplicity, it is assumed that the set $\mathcal{Y}$ to be inverted is a box $[\vec{y}]$. SIVIA uses the two following tests to decide whether a given box $[\vec{x}]$ is inside or outside the solution set $\mathcal{X}$ :

$$
\begin{aligned}
& \text { (i) } \forall i,[f]_{i}([\vec{x}]) \subset[y]_{i} \quad \Rightarrow[\vec{x}] \subset \mathcal{X} \\
& \text { (ii) } \exists i \mid[f]_{i}([\vec{x}]) \cap[y]_{i}=\emptyset \Rightarrow[\vec{x}] \cap \mathcal{X}=\emptyset .
\end{aligned}
$$

\section{Proofs}

(i) If $\forall i,[f]_{i}([\vec{x}]) \subset[y]_{i}$, then from $(3), \forall i, f_{i}([\vec{x}]) \subset[y]_{i}$, i.e. $\vec{f}([\vec{x}]) \subset[\vec{y}]$. Therefore $[\vec{x}] \subset \mathcal{X}$.

(ii) If $\exists i \mid[f]_{i}([\vec{x}]) \cap[y]_{i}=\emptyset$, then from (3), $\exists i \mid f_{i}([\vec{x}]) \cap[y]_{i}=\emptyset$.

Therefore $\vec{f}([\vec{x}]) \cap[\vec{y}]=\emptyset$, i.e. $[\vec{x}] \cap \mathcal{X}=\emptyset$.

SIVIA is a recursive routine that brackets the solution set $\mathcal{X}$ between an inner set of boxes and an outer set of boxes. For the sake of simplicity, it will be presented 
here in the case of a two dimensional solution set, but the algorithm readily extends to higher dimensions [8]. Boxes that have been proved to belong to $\mathcal{X}$ via test (i) are drawn in dark grey, those that have been proved to be outside $\mathcal{X}$ via test (ii) are drawn in light grey and those that satisfy neither (i) nor (ii) and are too small to be bisected are drawn in white. The accuracy $\varepsilon$ is a small positive real number.

\section{$\operatorname{SIVIA}(\vec{x})$}

Step $1 \forall i,[f]_{i}([\vec{x}]) \subset[y]_{i},\{\operatorname{draw}([\vec{x}]$, 'darkgrey'); return $\}$;

Step $2 \exists i \mid[f]_{i}([\vec{x}]) \cap[y]_{i}=\emptyset,\{\operatorname{draw}([\vec{x}]$, 'lightgrey'); return $\}$;

Step 3 If $w(\vec{x})<\varepsilon,\{$ draw $([\vec{x}]$, 'white'); return $\}$;

Step 4 Bisect $[\vec{x}]$ and store the two resulting boxes into $[\vec{x}](1)$ and $[\vec{x}](2)$;

Step $5 \quad \operatorname{SIVIA}([\vec{x}](1)) ; \operatorname{SIVIA}([\vec{x}](2))$;

SIVIA is first called for $[\vec{x}])=[\vec{x}](0)$, where $[\vec{x}](0)$ is a box assumed to contain the solution set $\mathcal{X}$. If we denote by $\Delta \mathcal{X}$ the union of all white boxes and by $\mathcal{X}^{-}$the union of all dark grey boxes, then the solution set $\mathcal{X}$ is bracketed by:

$$
\mathcal{X}^{-} \subset \mathcal{X} \subset \mathcal{X}^{-} \cup \Delta \mathcal{X}
$$


Remark 1 When the box $\mathcal{Y}$ to be inverted is a singleton $\{\vec{y}\}$ (for example when dealing with error-free data), the solution set $\mathcal{X}$ is often reduced to a singleton $\{\vec{x}\}$ which is easily found by SIVIA or by other punctual approaches. When two or more solutions exist, SIVIA detects all of them in a guaranteed way, contrary to punctual approaches.

Remark 2 Inversion methods generally considered are punctual: they try to find the best fit. In a linear context, numerical instability appears when the matrix to be inverted is almost non invertible. With a set inversion approach, the problem of instability does not exist. Even if the model is non identifiable (which corresponds to a situation where the matrix is non invertible in a punctual and a linear context), a set with a stretched shape is obtained. All informations about uncertainties (numerical, errors on measurements, ...) are given by $\mathcal{X}$ : if $\mathcal{X}$ is big, as in the application treated in the next section, the problem can be considered as "ill-posed" in a punctual point of view. 


\section{Results and discussion}

The experimental $\mathrm{CF}_{4}$ isotropic spectrum $I^{\text {iso }}$ reported in [5] had been recorded for a set of Raman frequency shifts $\omega_{i}$ relative to the green spectral line $\left(\lambda_{L}=\right.$ $514.5 \mathrm{~nm}$ ) of an argon laser. Following the bounded-error approach, uncertainties on experimental data are assumed to be bounded, i.e. the $i$ th ideal measurement $I_{i}^{i s o}$ (the measurement that should be obtained if no measurement errors occurred) is assumed to belong to the interval $\left[I_{\min }^{i s o}(i), I_{\max }^{i s o}(i)\right]$ provided in [5] and recalled in Table 1.

Possible location for Table 1

As regards the theoretical spectrum, and according to Eq. (1), the $i$ th model output is given by

$$
\begin{aligned}
I_{i}^{i s o}(A, E) & =D I D_{i}+\alpha^{2} A^{2} \alpha \mathbf{T A}_{i}+\alpha^{2} E^{2} \alpha \mathbf{T E}_{i} \\
& +A^{4} \mathbf{A T A}_{i}+A^{2} E^{2} \mathbf{A T E}_{i}+E^{4} \mathbf{E T E}_{i}
\end{aligned}
$$

The dipole-dipole and dipole-multipole components $\left(D I D_{i}=I_{D I D}^{i s o}\left(\omega_{i}\right), \alpha \mathbf{T A}_{i}=\right.$ $c_{\alpha \mathrm{TA}}^{i s o} I_{\alpha \mathrm{TA}}\left(\omega_{i}\right)$, etc.) are provided in Table 1 (see [5] for the details of their computation). Since the value of the $\mathrm{CF}_{4}$ polarizability is known $\left(\alpha=2.93 \AA^{3}\right.$ for 
$\left.\lambda_{L}=514.5 \mathrm{~nm}[12]\right)$, the unknown parameters are $A$ and $E$. Prior feasible intervals for $A$ and $E$ are given by

$$
A \in[0,2] \text { and } E \in[0,4] \text {. }
$$

where $A$ and $E$ are expressed in $\AA^{4}$ and $\AA^{5}$ units, respectively. Table 2 shows how the problem of estimating $A$ and $E$ can be cast into the framework of set inversion

\section{Possible location for Table 2}

In less than 5 seconds on a Pentium 100 Personal Computer, SIVIA brackets $\mathcal{S}$ as represented in Fig. 1.

\section{Possible location for Figure 1}

Because of some mechanism not taken into account in our semi-classical model, it may happen that SIVIA eliminates a part of the parameter space that could contain the true values of $A$ and $E$. To protect against this, one would like to be especially careful about data points that turn out to have a critical influence on the size of $\mathcal{S}$. 
For this purpose, we define the safety of the $i$ th interval data by

$$
\gamma(i)=\frac{\operatorname{Vol}(\mathcal{S})}{\operatorname{Vol}\left(\mathcal{S}_{i}\right)}
$$

where $\mathrm{Vol}$ corresponds to the volume (an area in our two-dimensional case) and $\mathcal{S}_{i}$ is the set of all parameters that are consistent with all data but the $i$ th. The smaller the safety is, the more careful one must be with the corresponding data point. SIVIA can easily be adapted to compute volumes of sets [8] and therefore the coefficients $\gamma(i)$. We obtain $\gamma(1)=\gamma(3)=\gamma(5)=1$., which means that any of the associated data points can be removed without changing the feasible domain for $(A, E)$. Moreover, $\gamma(2)=0.98, \gamma(4)=0.98, \gamma(6)=0.93$ and $\gamma(8)=0.74$. The safety of the 8th measurement is the smallest and the reliability of the semi-classical model at the corresponding frequency $\omega_{8}=120 \mathrm{~cm}^{-1}$ can be questioned.. After removing the 8th measurement, SIVIA brackets $\mathcal{S}_{8}$ as represented in Fig. 2. Note that the upper boundary of $\mathcal{S}_{8}$ is not significantly different from that of $\mathcal{S}$, but the lower boundary of $\mathcal{S}_{8}$ lie much lower.

\section{Possible location for Figure 2}

In [5], an empirical estimation leads to the intervals $[0.5,1.2] \AA^{4}$ and $[1.0,3.5] \AA^{5}$ for $A$ and $E$, respectively. The corresponding rectangle, drawn with dashed lines in Figs.1- 
2, contains a large part of the solution sets $\mathcal{S}$ and $\mathcal{S}_{8}$. Moreover, the maximum values of $A$ and $E$ given in [5] are very close to those provided here. The aforementioned rectangle, however, also contains parameter vectors that are not consistent with the data. Finally, note that the vector $(A, E)=\left(0.97 \AA^{4}, 1.15 \AA^{5}\right)$, recently calculated $a b$ initio by Maroulis $[9,10]$ and represented by a white cross on Figs. 1-2, is located outside $\mathcal{S}$ and deep inside $\mathcal{S}_{8}$. This corroborates the hypothesis that the 8 th data is not consistent with the semi-classical model. The discrepancy may be due to short range effects that take place for high frequencies of each dipole-multipole contribution. When scattering data for frequency shifts higher than $120 \mathrm{~cm}^{-1}$ are considered, similar discrepancies can be observed [5].

In conclusion, the set inversion approach, advocated here, makes it possible to estimate unknown parameters, their uncertainties, as well as their correlations, when bounded-error data and nonlinear models are involved. Such problems arise often in spectroscopy where many efforts are focused on estimating physical coefficients from experimental data (e.g. collision-induced scattering or absorption processes). The example of recent CIS studies on gaseous $\mathrm{CF}_{4}$ is but one illustration of the advantages of set-inversion over more conventional methods. 


\section{References}

[1] Collision-and Interaction-Induced Spectroscopy, vol. 452 of NATO ASI Series C: Mathematical and Physical Sciences Edited by G.C. Tabisz and M.N. Neuman, Kluwer Academic Publishers, Dordrecht (1995).

[2] A. D. Buckingham and G. C. Tabisz, Mol. Phys. 36, 583 (1978).

[3] H. Posch, Mol. Phys. 46, 1213 (1982).

[4] A. Elliasmine, J.-L. Godet, Y. Le Duff, and T. Bancewicz, Mol. Phys., 90, 147 (1997).

[5] A. Elliasmine, J.-L. Godet, Y. Le Duff, and T. Bancewicz, Phys. Rev. A, 55, (June 1997).

[6] E. Walter and L. Pronzato, Identification of Parametric Models from Experimental Data, Springer, London, (1997).

[7] Bounding Approaches to System Identification, Edited by M. Milanese, John Norton, H. Piet-Lahanier and E. Walter, Plenum, New York (1997).

[8] L. Jaulin and E. Walter, Automatica, 29, 1053 (1993).

[9] G. Maroulis, Chem. Phys. Lett., 259, 654 (1996) 
[10] G. Maroulis, J. Chem. Phys., 105, 8467 (1996).

[11] R.E. Moore, Methods and Applications of Interval Analysis, SIAM, Philadelphia, 1979.

[12] H. E. Watson and K. L. Ramaswamy, Proc. Roy. Soc. London A 156, 144 (1936). 
Table 1

Experimental intensities of the $\mathrm{CF}_{4}$ isotropic spectrum versus frequency shifts together with corresponding dipole-dipole and dipole-multipole theoretical contributions.

\begin{tabular}{|c||c|c|c|c|c|c|c|c||c|}
\hline$i$ & 1 & 2 & 3 & 4 & 5 & 6 & 7 & 8 & units \\
\hline \hline$\omega$ & 50 & 60 & 70 & 80 & 90 & 100 & 110 & 120 & $\mathrm{~cm}^{-1}$ \\
\hline $\begin{array}{c}I_{\text {min }}^{\text {iso }} \\
I_{\text {max }}^{\text {iso }}\end{array}$ & 192 & 177 & 116 & 75 & 41 & 22 & 15 & 10 & $10^{-58} \mathrm{~cm}^{6}$ \\
\hline DID & 1003 & 656 & 299 & 165 & 89 & 61 & 41 & $10^{-58} \mathrm{~cm}^{6}$ \\
$\alpha$ TA & 133.1 & 80.57 & 44.55 & 22.55 & 10.47 & 4.53 & 1.87 & 0.77 & $10^{-58} \mathrm{~cm}^{6} \AA-14$ \\
$\alpha$ TE & 6.39 & 4.78 & 3.39 & 2.27 & 1.44 & 0.87 & 0.50 & 0.27 & $10^{-58} \mathrm{~cm}^{6} \AA^{-16}$ \\
ATA & 196.1 & 153.7 & 114.9 & 81.9 & 55.7 & 36.1 & 22.4 & 13.3 & $10^{-60} \mathrm{~cm}^{6} \AA^{-16}$ \\
ATE & 20.20 & 16.94 & 13.73 & 10.74 & 8.12 & 5.93 & 4.19 & 2.86 & $10^{-60} \mathrm{~cm}^{6} \AA^{-18}$ \\
ETE & 0.70 & 0.61 & 0.52 & 0.43 & 0.35 & 0.27 & 0.21 & 0.16 & $10^{-60} \mathrm{~cm}^{6} \AA^{-20}$ \\
\hline
\end{tabular}




\section{Table 2}

Translation table between the set inversion formalism and the problem of estimating the $A$ and $E$ values of $\mathrm{CF}_{4}$.

\begin{tabular}{|c|c|}
\hline Set inversion & Estimation of $A$ and $E$ \\
\hline \hline$[y]_{i}$ & {$\left[I_{\min }^{\text {iso }}(i), I_{\max }^{\text {iso }}(i)\right]$} \\
\hline $\mathcal{Y}=[\vec{y}]$ & {$\left[I_{\min }^{\text {iso }}(1), I_{\max }^{\text {iso }}(1)\right] \times \cdots \times\left[I_{\min }^{\text {iso }}(8), I_{\max }^{\text {iso }}(8)\right]$} \\
\hline$\vec{x}$ & $(A, E)^{T}$ \\
\hline$f_{i}(\vec{x})$ & $I_{i}^{m}(A, E)$ \\
\hline$\vec{f}$ & $\left(I_{1}^{m}(A, E), \cdots, I_{8}^{m}(A, E)\right)^{T}$ \\
\hline $\mathcal{X}=\{\vec{x} \mid \vec{f} \subset \mathcal{Y}\}$ & $\mathcal{S}=\left\{(A, E) \mid \forall i, I_{i}^{m}(A, E) \in\left[I_{\text {min }}^{i s o}(i), I_{\text {max }}^{\text {iso }}(i)\right]\right\}$ \\
\hline$[\vec{x}](0)$ & {$[0,2] \times[0,4]$} \\
\hline
\end{tabular}




\section{Figure Captions}

Figure 1: Set of $A$ and $E$ that are consistent with all data (dark grey area) in the $(A, E)$ plane. The units are in $\AA^{4}$ for $A$ and in $\AA^{5}$ for $E$. The dashed rectangle corresponds to the intervals for $A$ and $E$ provided in [5], the white cross represents the vector $(A, E)$ found by Maroulis [9, 10], and the light grey area is the set of forbidden parameter vectors.

Figure 2: Set of $A$ and $E$ that are consistent with the first seven data. 


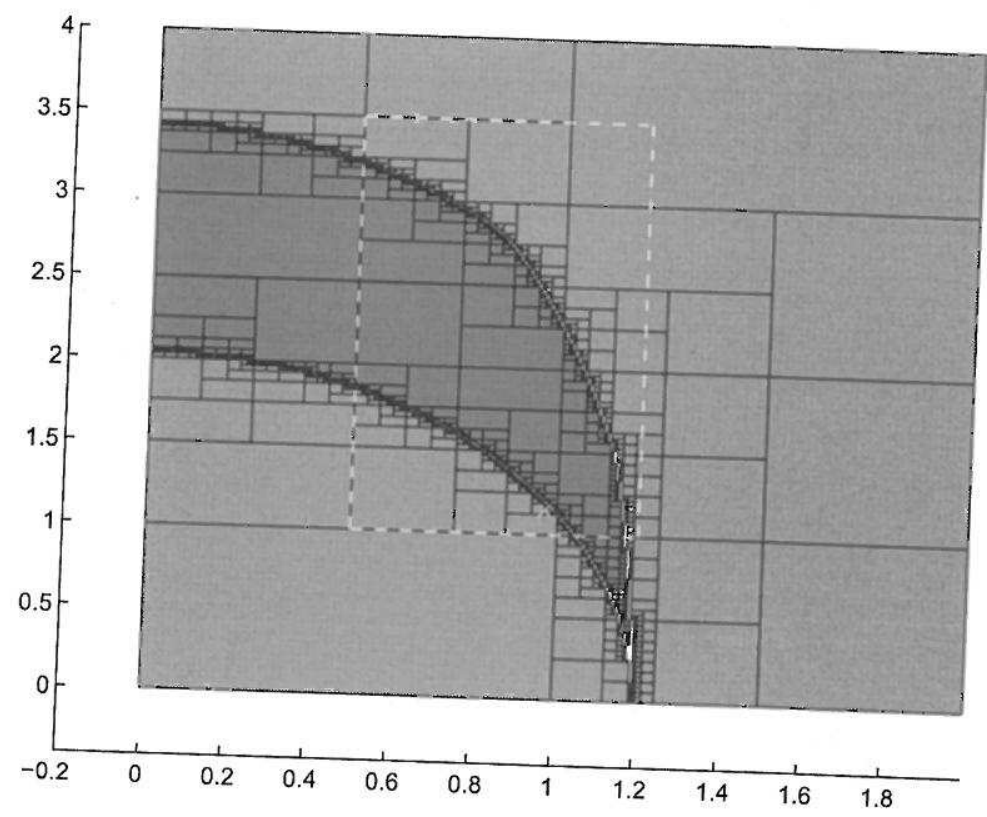

Figure 1: 


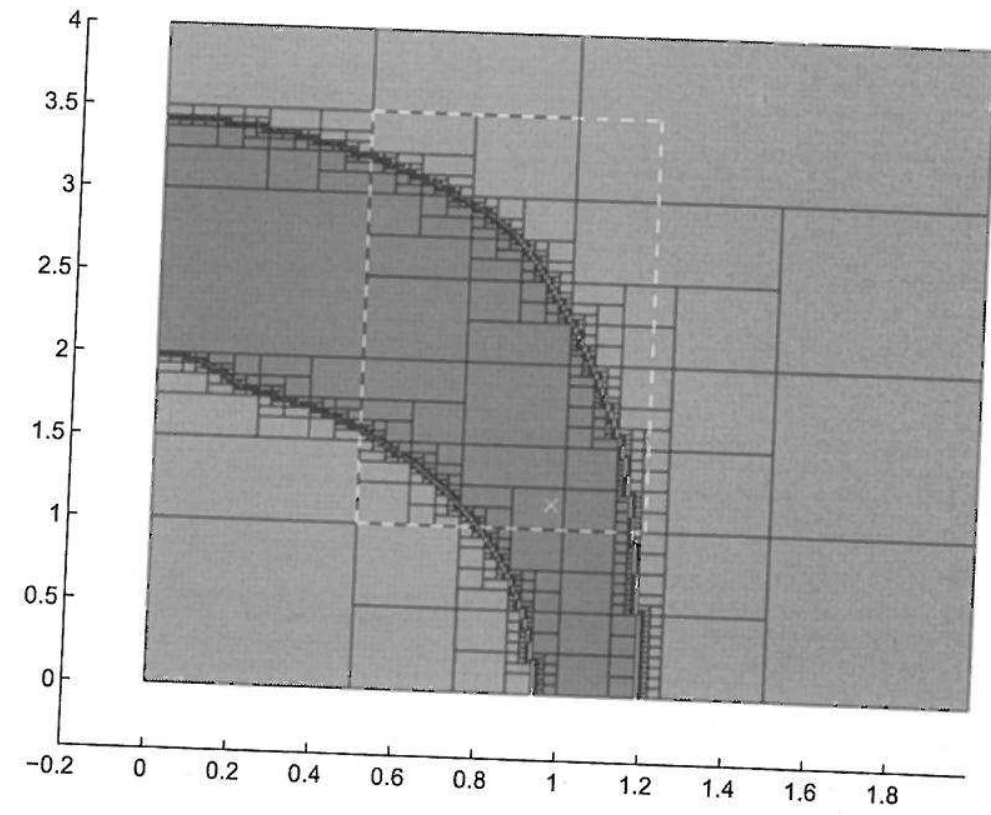

Figure 2: 